\title{
Buen vivir, economías solidarias y universidades públicas en Perú
}

Luis Montoya-Canchis*

Magíster en Sociología Política. Profesor, Departamento Académico de Sociología, Facultad de Ciencias Sociales, Universidad Nacional Mayor de San Marcos, Lima, Perú.

Correo electrónico:

lmontoyac@unmsm.edu.pe

Recibido: 17 de enero del 2016 Aceptado: 31 de mayo del 2016

Cómo citar este artículo: MontoyaCanchis, L. (2016). Buen vivir, economías solidarias y universidades públicas en Perú. Cooperativismo \& Desarrollo, 24(109), xx-xx. doi: 10.16925/co.v24i109.1506

\section{Resumen}

Propósito: el trabajo expone la experiencia del Seminario de Economía Social, Solidaria y Popular de la Universidad Nacional Mayor de San Marcos, de Perú. Descripción: asumir el desafío de refundar las universidades e imaginar nuevos programas de acción universitarios exige cuestionar la imposición de la estructura de saber eurocéntrico con el fin de apuntar hacia su decolonización y, por eso, promover diálogos entre el saber científico y humanístico, así como con los saberes legos, populares, tradicionales, urbanos, de culturas no occidentales. Punto de vista: se busca superar la división académica por disciplinas para proponer una estructuración inter y transdisciplinaria, y lograr que la formación profesional, la investigación dirigida a atender las demandas de la sociedad capitalista y la administración pública que le corresponde den paso a otra orientada por el buen vivir, las economías solidarias, la socialización y descolonización del saber, además de las relaciones de poder que suponen un gran reto. Conclusiones: a través de este recorrido se pretende desenvolver un diálogo de saberes sobre las economías sociales, solidarias, populares, comunitarias, así como experiencias de intervención y procesos de incidencia en el ámbito universitario.

Palabras clave: buen vivir, economía solidaria, Perú, universidad pública. 


\title{
Good living, solidarity economies, and public universities in Peru
}

\begin{abstract}
Purpose: This paper presents the experience of the Social, Solidarity, and Popular Economies Seminar at the Universidad Nacional Mayor de San Marcos in Peru. Description: Taking up the challenge of reshaping universities and imagining new university programs of action demands that we question the imposition of the Eurocentric structure of knowledge. This is necessary in order to promote its decolonization and, therefore, encourage dialogue between scientific and humanistic knowledge, as well as with lay, popular, traditional, and urban knowledge from non-Western cultures. Viewpoint: An attempt is made to overcome the academic division of disciplines in order to propose an inter- and transdisciplinary structuring. This structuring will then make professional training, research aimed at meeting the demands of capitalist society, and the necessary public administration give way to another one oriented toward good living, solidarity economies, and the socialization and decolonization of knowledge, in addition to the power relations that pose a great challenge. Conclusions: This review is intended to develop a dialogue of knowledges about the social, solidarity, popular, and community economies, as well as experiences of intervention and advocacy processes at the university level.
\end{abstract}

Keywords: good living, solidarity economy, Peru, public university

\section{Bom viver, economias solidárias e universidades públicas em Peru}

\section{Resumo}

Propósito: este trabalho expõe a experiência do Seminário de Economia Social, Solidária e Popular da Universidad Nacional Mayor de San Marcos, do Peru. Descrição: assumir o desafio de reformular as universidades e imaginar novos programas de ação universitários exige questionar a imposição da estrutura de saber eurocêntrico com o fim de apontar para sua decolonização e, por isso, promover diálogos entre o saber científico e humanístico, bem como com os saberes leigos, populares, tradicionais, urbanos, de culturas não ocidentais. Ponto de vista: procura-se superar a divisão acadêmica por disciplinas para propor uma estruturação inter e transdisciplinar, e conseguir que a formação profissional, a pesquisa dirigida a atender as demandas da sociedade capitalista e a administração pública que lhe corresponde deem passo a outra, orientada pelo bom viver, pelas economias solidárias, pela socialização e descolonização do saber, além das relações de poder que supõem um grande desafio. Conclusões: por meio deste percurso, pretende-se desenvolver um diálogo de saberes sobre as economias sociais, solidárias, populares, comunitárias, bem como experiências de intervenção e processos de incidência no âmbito universitário.

Palavras-chave: bom viver, economia solidária, Peru, universidade pública. 


\section{Introducción}

¿La producción de saberes generados en el buen vivir ${ }^{1}$ y las economías solidarias ${ }^{2}$, animada desde las diversas organizaciones y los movimientos sociales, así como las comunidades urbanas y rurales de muchos lugares de Latinoamérica y el mundo constituye una variable determinante de los procesos de desenvolvimiento de estas?

Quijano (2011b) indica con pertinencia que la explotación consiste en el control sobre la fuerza de trabajo y lo producido, lo cual no puede hacerse sin dominación; pero la dominación no incluye solo el uso de la autoridad, de la fuerza y su posterior naturalización y legitimación, sino también el control de la subjetividad, del conocimiento y del modo de producir conocimiento. Los saberes de las personas involucradas en las diversas experiencias de buen vivir y economías solidarias constituyen un patrimonio inmaterial al cual se puede apelar para persistir en el establecimiento de una manera diferente de organizar las relaciones económicas y sociales, de poder, simbólicas, con la naturaleza de la sociedad moderna colonial capitalista.

Sin embargo, estos saberes no son reconocidos e incluso son invisibilizados. En el mejor de los casos, se consideran parte del sentido común y se ubican en un canon epistemológico académico universitario eurocéntrico en un nivel inferior.

¿Esta perspectiva sobre al saber producido, propia de la defensa por el buen vivir y las economías solidarias, es hegemónica? ¿Existen perspectivas

El buen vivir, según Quijano (2011a), es una propuesta proveniente del nuevo movimiento de los indígenas latinoamericanos, que, para ser una realización histórica efectiva, es un complejo de prácticas sociales orientadas a la producción y a la reproducción democráticas de una sociedad democrática, un otro modo de existencia social, con su propio y específico horizonte histórico de sentido, radicalmente alternativos a la colonialidad global del poder y a la colonialidad, moderna europea. Hoy solo puede tener sentido como una existencia social alternativa, como una des-colonialidad del

2 La economía social y solidaria es, siguiendo a Coraggio (2011), una forma de producir sociedad y no solo utilidades económicas, porque genera valores de uso para satisfacer necesidades de los mismos productores o de sus comunidades -generalmente de base territorial, étnica, social o cultural- y no está orientada por la ganancia y la acumulación de capital sin límites. Vuelve a unir producción y reproducción, al producir para satisfacer de manera más directa y mejor las necesidades acordadas como legítimas por la misma sociedad [...] Su fundamento es, sin duda, el trabajo y el conocimiento encarnado en los trabajadores y sus sistemas de organización (pp. 45-46). alternativas? ¿Las universidades mantienen inalterable el canon epistemológico académico universitario de carácter eurocéntrico? ¿Existen experiencias que muestren evidencias de vinculaciones diferentes?

Queremos centrarnos en el examen de una de las instituciones directamente ligadas a la producción de conocimiento: las universidades. Para este ejercicio exploratorio analizaremos el Seminario de Economía Social, Solidaria y Popular de la Universidad Nacional Mayor de San Marcos, de Lima, Perú. Este caso nos permitirá mostrar la complejidad de una historia y memoria construida, las tensiones internas desenvueltas, junto a sus desafíos y perspectivas de futuro. Nos centramos en este caso por la accesibilidad a la información que disponemos y porque el Perú, a diferencia de otros países de Latinoamérica, representa un lugar donde es casi nulo el interés del Estado por estos asuntos.

\section{Un viejo teatro como punto de encuentro}

La discusión sobre las relaciones entre buen vivir, las economías solidarias y las universidades no es nueva en la reflexión latinoamericana. Uno de los que propiamente abre las condiciones para su tratamiento a comienzos del siglo xxi es Coraggio. Su crítica al economicismo-utilitarismo propio del planteamiento neoliberal sustenta una lectura alternativa sobre las universidades y sus relaciones con la sociedad y la economía.Esto abre la posibilidad de nuevos desafíos hacia el futuro. Él señala al respecto, cuando aborda la relación entre las universidades y lo local:

[...] implica tener una propuesta de universidad que entre en confrontación directa con el programa de su disolución a manos del mercado. Implica la recomposición del sistema universitario para contribuir a la generación de utopías, la crítica epistemológica de las verdades desde las cuales se justifican las políticas de dominio, el desarrollo de estrategias deinvestigación y formación que contribuyan a la constitución de actores sociales y políticos democráticos (Coraggio, 2002, p. 10) ${ }^{3}$.

3 Es pertinente mencionar que antes de la existencia de lecturas críticas como las de Coraggio, existió una larga, extendida y diversa suma de esfuerzos centrados en la relación entre universidades y cooperativas en muchos lugares de Latinoamérica, lo que 
El propio Coraggio, en otro texto escrito años después, precisa sobre la relación entre economías sociales y solidarias, y universidades:

La perspectiva de una economía social y solidaria da lugar a un programa de acción estratégica para la transición, basado en prácticas micro, meso y sistémicas con dimensiones sociales, culturales y políticas, que requiere la transformación de una serie de instituciones que una mirada economicista afirmaría que no son parte del sistema económico (de mercado) a menos que justamente pauten la organización económica como negocios privados [...] Una de esas instituciones es la universidad, una forma histórica de pautar la producción y distribución del conocimiento "racional", como activo y como potencialidad, en ambos casos de fuerte y creciente efectividad en el desempeño de las economías. De hecho, las universidades y centros de investigación han estado traccionadas durante mucho tiempo hacia la demanda (sostenida pecuniariamente) de los grandes negocios y el Estado neoliberal tanto en cuanto a la formación de profesionales como a la investigación sobre problemas que definen como prioritarios, porque obstaculizan el crecimiento de capitales privados o de la economía nacional (Coraggio, 2014, p. 4).

Coraggio comparte la tesis de que para que se dé una revolución en el sistema universitario (en la formación, la investigación, los servicios y la acción conjunta con la comunidad) influida por las economías sociales y solidarias se debe revisar las características fundantes de la modernidad de las cuales la universidad es heredera. Esto lleva a dejar de reproducir y sostener, a pesar de sus fracasos, el paradigma del desarrollo y la modernización, lo que da cabida a la decolonialidad del poder y el saber, así como a propuestas descolonizadoras como el buen vivir ${ }^{4}$.

Pastore (2010) llama "la economía social tradicional", tanto desde la perspectiva de la implementación de programas de formación en cooperativismo, experiencias de extensión universitaria sobre todo rural o y investigaciones dedicadas a las cooperativas, la participación de los trabajadores y la autogestión obrera.

4 Este aspecto en particular es trabajado con agudeza por Germaná (2013) cuando señala que para los dominados y explotados la decolonialidad de la universidad es el cuestionamiento radical a la colonialidad de la universidad. Este cuestionamiento pasa por tres principios: primero, la superación de la hegemonía de las estructuras eurocéntricas del saber; segundo, la superación de las estructuras
Sin embargo, Coraggio (2014) también comparte una evaluación crítica de las relaciones establecidas entre los universitarios y las universidades con los actores de las economías sociales y solidarias. Según él, estas han sido "limitadas a intervenciones marginales y orientadas moralmente (extensión universitaria, mayormente estudiantes socialmente comprometidos, con tendencias al paternalismo)" (p. 5). Agrega además que "también se atienden las demandas mediadas por contratos públicos de servicios de formación e investigación, en buena medida dirigidas al diseño, gestión y evaluación de políticas sociales asistencialistas focalizadas en la pobreza extrema" (p. 5).

Esta crítica nos parece aguda y provocadora. Responder con un argumento antitético u oponernos simplemente no nos permitiría mostrar la complejidad característica de las relaciones entre el buen vivir, las economías solidarias y las universidades.

No podemos dejar de mencionar, en primer lugar, que las universidades de Latinoamérica poseen una larga historia y, en más de un caso, anteceden al propio Estado, por lo tanto su tratamiento no debería ser simplificado. Todo lo contrario constituyen un viejo teatro, un punto de encuentro entre actores diversos, de procesos y tendencias determinadas desde libretos escritos muchas veces desde fuera o más allá de la universidad, enmarcados en estructuras ancladas local, nacional y hasta globalmente. Por lo tanto, es necesario reconocer a los protagonistas de las tramas que serán interpretadas en este teatro, conocer sus argumentos, apreciar sus interpretaciones; de lo contrario corremos el riesgo de asumir que estamos siendo espectadores de una historia con un desenlace previsible y, peor aún, con un final previamente determinado.

En segundo lugar, los actores de las experiencias del buen vivir y de las economías solidarias tampoco constituyen sectores pasivos o caracterizados por reunir actores sin iniciativa o desmotivados de relacionarse con otros actores y construir su propios derroteros. Todo lo contrario hay dinamismo en los procesos que se desenvuelven en el buen vivir y las economías solidarias, y puede haber relaciones en diferentes planos. Incluida está la posibilidad de nuevas perspectivas en los vínculos tejidos por los actores

institucionales disciplinarias, y, tercero, por el diálogo entre el saber académico y los saberes de los grupos sociales subalternizados por la colonialidad del poder. Castro-Gómez (2007) aborda también este asunto y propone que "decolonizar la universidad", lo cual favorece la transdisciplinariedad y la transculturalidad. 
del buen vivir y las economías solidarias con las universidades, y entre ellos y la sociedad.

El supuesto de soporte del cual partimos es que las universidades no responden solo a una determinación externa del Estado, la sociedad o el mercado, sino además son resultado de la acción los actores universitarios: autoridades, docentes, juventudes universitarias, trabajadores no docentes, graduados. Además hay un contexto de acelerados cambios globales que suponen nuevas relaciones y ámbitos de acción para las universidades. Así como existe una reconfiguración de los actores con los cuales las universidades, en mayor o menor medida, se relacionan. Por lo tanto, sus expectativas, demandas reales o potenciales, reivindicaciones programáticas o agendas de acción colectiva experimentan también cambios profundos y generan nuevos desafíos para estas ${ }^{5}$.

¿Este viejo teatro, entonces, es realmente un punto de encuentro con el buen vivir y las economías solidarias? Apelando una vez más a Coraggio (2014), podemos señalar:

Un claro ejemplo de la inadecuación e inflexibilidad del sistema universitario respecto a las demandas directamente sociales, es el reciente recurso de los movimientos indígenas a crear sus propias instituciones de formación e investigación, aún por fuera del sistema de reconocimiento formal de las capacidades de los que participen de esos procesos. A la vez, los profesionales para el sector público no son formados para establecer una relación dialógica entre saberes con los sujeto-objetos de las intervenciones estatales, reproduciendo la cultura de "expertos" propia de la Modernidad, hoy bajo crítica (p. 5).

Como se ve, para Coraggio, las posibilidades de que las universidades puedan convertirse en ejemplos del buen vivir y las economías solidarias son restringidas. Hay una serie de aspectos que conspira en contra de la idea de la necesaria articulación de las universidades con proyectos de transformación civilizatorias, de la vida y los sistemas económicos, como el buen vivir y las economías solidarias.

5 Al respecto coincidimos con una afirmación de Leher (2010): "[l] as reconfiguraciones en curso en las instituciones universitarias son de tal magnitud que resulta un error insistir en que el problema está relacionado tan solo con la presencia o la ausencia del Estado en la educación superior" (p. 20).
A pesar de esto, Coraggio permite la posibilidad de encontrar un elemento clave que coadyuve a revertir esta situación. Él señala al respecto:

Es preciso que haya sujetos colectivos comunitarios y sociales que planteen sus necesidades de formación, conocimiento y servicios en una relación dialógica institucionalizada pero abierta a la innovación y el aprendizaje. Esto puede permearse al conjunto de la universidad que, sin embargo usualmente se resistirá burocráticamente (Coraggio, 2014, p. 5).

Entonces, no está cerrada del todo la posibilidad de convertir a las universidades en casos de buen vivir y en economías solidarias, siempre y cuando siguiendo el planteamiento de Coraggio- existan estos sujetos colectivos comunitarios y sociales. Lo interesante, desde nuestro punto de vista, es que estamos otra vez confrontando con un planteamiento que recupera el peso de los actores, pero, en este caso, pensados desde fuera de las universidades. Si llamábamos la atención sobre la necesidad de reconocer a los actores de las universidades, Coraggio advierte sobre la necesidad de reconocer en la sociedad a los actores que potencialmente pueden interpelar a las universidades y provocar nuevos procesos que redefinan sus vínculos con la sociedad. Este abordaje nos parece mucho más conveniente metodológicamente para el tratamiento de las relaciones entre buen vivir, las economías solidarias y las universidades, porque nos enfrenta con la necesidad de identificar a los actores, indagar en las relaciones que desenvuelven, analizar sus discursos, recuperar sus memorias, antes que presuponer que las estructuras económicas, sociales, culturales o políticas constituidas han determinado y determinan todo. Nos desafía de manera expresa, debido a que abre la posibilidad de escribir una historia donde el final no está previamente determinado.

No es que desconozcamos el peso que las estructuras poseen, sobre todo en el caso de dinámicas institucionales como de las universidades, pero es necesario ir más allá de los condicionamientos estructurales y acercarse a una historia, que sin negar los determinantes generados por las estructuras y las dinámicas económico sociales, culturales y político institucionales pueda incluir a los actores del buen vivir, las economías solidarias y las universidades. Esta perspectiva centrada en el examen de las relaciones entre estructuras y actores permite indagar en ambos polos, así como una perspectiva más integral de las relaciones establecidas entre ambos. 
Un aspecto necesario para sumar en la discusión, y que adquiere particular transcendencia, tiene que ver con lo que Germaná llama "el carácter monocultural de las universidades" y el imperativo de que estas se conviertan en interculturales. Es decir -según sus propias palabras- en espacios:

Donde dialoguen en igualdad de condiciones las diversas tradiciones culturales, tanto las que han sido hegemónicas en el patrón de dominación moderno/ colonial, como las perspectivas de conocimiento que habían sido reprimidas o excluidas por este patrón de dominación social. Se trata del principio de la "ecología de saberes" que propone Boaventura de Sousa Santos, el que "consiste en la promoción de diálogos entre el saber científico y humanístico que la universidad produce y los saberes legos, populares, tradicionales, urbanos, campesinos, provincianos, de culturas no occidentales (indígenas de origen africano, oriental, etc.) que circulan en la sociedad (citado por Germaná, 2013, p. 17).

Las universidades latinoamericanas poseen predominantemente un carácter monocultural, porque buscan en su mayoría reproducir el patrón de dominación moderno colonial eurocéntrico, pero afrontan tensiones profundas con sus sociedades, caracterizadas por la heterogeneidad estructural y cultural ${ }^{6}$. Las universidades actúan muchas veces como enclaves que reclutan jóvenes que adquieren la condición de universitarios a través de un proceso de formación que en general los termina alejando de sus comunidades y lugares de origen. Peor aun los universitarios contraponen de manera expresa los saberes que portan y heredan de sus familias con los saberes de las comunidades científicas existentes dentro de las universidades, estableciendo jerarquías con base en modelos y estereotipos foráneos; esto ocasiona su desvinculación y desarraigo con los lugares donde nacieron, y reproduce mecanismos de dependencia cultural, científica y tecnológica. Este conjunto de elementos entran en contraposición con la

\footnotetext{
6 Nos parece necesario ubicar estas tensiones como parte del conflicto entre tendencias de reoriginalización cultural y represión/ reabsorción, que Quijano (1997) considera han impregnado nuestra más profunda experiencia histórica, porque no solamente subyace a nuestros problemas de identidad, sino que atraviesa todas nuestra historia, desde el comienzo mismo de la constitución de América, como una tensión continua de la subjetividad, donde el carácter del imaginario y de los modos de conocer y de producir conocimiento son una cuestión abierta y conflictiva.
}

demanda de reconocimiento creciente que reclaman las varias tradiciones culturales, y los saberes que poseen y reproducen, existentes en las sociedades latinoamericanas.

Esta situación de tensión exige afirmar procesos de diálogo intercultural, tanto dentro como fuera de las universidades, los cuales no están exentos de debilidades o conflictos que evidentemente dificultan su afianzamiento en el corto plazo; al mismo tiempo aportan a la generación creciente de corrientes de opinión favorables a la diversidad y el respeto por las diferencias desde las universidades. Estos componentes pueden ser cruciales para la relación entre la propuesta y las experiencias de buen vivir y las economías solidarias, las cuales son portadoras de saberes otros, diversos y necesarios de ser valorados, al menos, desde una perspectivas interesada en el desenvolvimiento de un diálogo de saberes descolonizador de las universidades.

Este asunto plantea el desafío de ir más allá del principal producto de la reforma universitaria surgida a partir de grito de Córdoba, en 1918; ; lo que Germaná describe del siguiente modo:

La universidad moderna/colonial. Abandonar la reforma universitaria y avanzar hacia la refundación de las universidades como instituciones exige nuevos programas de acción universitaria. Tres características principales -siguiendo a Germaná- definieron el modelo de universidad moderna colonial: La imposición de las estructuras de saber eurocéntricas, como perspectiva hegemónica de producir conocimientos; la institucionalización del saber en estructuras académicas disciplinarias; $y$, finalmente, el posicionamiento de las funciones de formación profesional de la mano de obra calificada y de la investigación para el capitalismo periférico y la administración pública. (2013, p. 7).

Asumir el desafío de refundar las universidades e imaginar nuevos programas de acción universitario exige cuestionar la imposición de la estructura de saber eurocéntrico con el fin de apuntar hacia su descolonización y, por ello, a la promoción de diálogos entre el saber científico y humanístico, así como con los saberes legos, populares, tradicionales, urbanos,

$7 \quad$ Es pertinente anotar que la movilización juvenil universitaria, de comienzos del siglo $\mathrm{xx}$, no solo surge en Córdoba sino tiene como antecedente el levantamiento de Cusco en 1909, como Rénique (1987) indica. 
de culturas no occidentales. También se propone superar la división académica por disciplinas, para proponer una estructuración inter y transdisciplinaria; disputar la formación profesional y de investigación dirigida a atender las demandas de la sociedad capitalista y la administración pública propia de esta, por otra orientada al buen vivir, las economías solidarias, la socialización y descolonización del saber y las relaciones de poder.

\section{Actores escribiendo su libreto}

\section{Situación en Latinoamérica}

Es necesario empezar señalando que las experiencias de vinculación entre la propuesta del buen vivir, propia de los pueblos originarios latinoamericanos, y las universidades se animan recientemente desde los primeros años de la década del noventa del siglo $\mathrm{xx}$. La reflexión de intelectuales de estos pueblos sumada a la creación de universidades indígenas son su principal expresión. La Universidad de las Regiones Autónomas de la Costa Caribe Nicaragüense, creada en 1992, figura entre las primeras (Mato, 2011, p. 73).

Sin embargo, también puede mencionarse como un antecedente a la Universidad Autónoma Indígena de México, de Mochicahui, El Fuerte, Sinaloa, iniciada en 1982, como un programa de investigación antropológica con aplicación en investigación y docencia, para indígenas de México y Latinoamérica o mestizos (Padilla, 2008, p. 52).

La relación entre las economías solidarias y las universidades, por su parte, es más antigua. Incluso reúne en algunos países una historia larga, como el caso de Argentina, donde existió un programa de formación universitario en cooperativismo que otorgaba una licenciatura en la Universidad Nacional de La Plata entre 1953 y el 2002, el primero de Latinoamérica; este luego se convirtió en tecnicatura (Acosta y Levin, 2013, p. 7).

Venezuela es otro caso que muestra las relaciones entre cooperativas y universidades que, como en la Universidad de Los Andes de Mérida, poseen antecedentes que se remontan por lo menos a los años sesenta. Estas relaciones son desenvueltas desde la creación del Centro de Estudios Cooperativos, fundado en 1963 (García, 2007, p. 17).

En México se dio el caso de la Universidad Autónoma de Querétaro, la cual desde 1969 forma técnicos en cooperativas. Esta enseñanza se eleva a carrera universitaria a partir del 2009, bajo el nombre de gestor de empresas sociales (Izquierdo, 2009, p. 116).

Estas vinculaciones no solo incluyen a las cooperativas o las organizaciones de las economías sociales tradicionales, sino también a experiencias más recientes como las de las empresas recuperadas, que, como en el caso de Brasil, han establecido relaciones con universidades, a través de las llamadas Incubadoras Tecnológicas de Cooperativas Populares (ITCP), creadas desde mediados de los años noventa y que a la fecha suman setenta experiencias en todo el país (Cruz, Tillman, Della Vechia y Nunes, 2011).

\section{El caso de Perú}

El caso específico del Perú permite registrar evidencias principalmente de vínculos entre economías solidarias y universidades desde varias décadas atrás, así como una casi inexistente presencia de la propuesta del buen vivir entre estas.

Anotamos que en el caso peruano, como adelantamos en la introducción, asuntos como el buen vivir y las economías solidarias no son un tema visible ni mucho menos central de la agenda pública. No debemos olvidar el contexto de aplicación de políticas de liberalización de mercados, caracterizado por ser particularmente duro e inflexible, desplegado desde comienzos de los noventa de la mano de un régimen autoritario como el liderado por el presidente Fujimori, que llevó a la legitimación de un discurso extremo pro mercado sin atenuantes en el conjunto de la sociedad peruana, y desestimó cualquier otro discurso diferente de la vida y la economía.

Sin embargo, es posible registrar antecedentes en el Perú de los vínculos entre economías solidarias y universidades, particularmente con las cooperativas, desde los sesenta. Esto ocurre por lo menos en dos universidades: la Universidad Particular San Martin de Porres y la Universidad Nacional Federico Villareal. En estas existieron programas dirigidos a la formación profesional en cooperativismo y actividades de extensión universitaria con cooperativas desde 1966 (Cárdenas, 1983, pp. 356-359).

Lo paradójico es que en paralelo al contexto generado por las políticas de liberalización de mercados se produjo lo que Mogrovejo, Vanhuynegem y

8 Anotamos que existe amplia literatura dedicada a estas experiencias: los primeros trabajos en los se indaga en ellas fueron escritos a comienzos del 2000, como los textos de Singer (2011) y Cruz (2004) 
Vásquez (2012), llaman el "renacimiento del modelo cooperativista peruano", caracterizado por el resurgimiento de un amplio y diverso número de experiencias cooperativistas, especialmente, en el sector agrario, vinculado al mercado global y dedicado a la exportación de productos como el café.

Por esto, los pocos esfuerzos que hubo para vincular economías solidarias, principalmente cooperativas y universidades, sobre todo en el campo de la formación y la extensión universitaria, fueron debilitados o desaparecieron en el marco del contexto adverso de aplicación de las políticas de liberalización de mercados. Hoy existe solo un posgrado universitario en el conjunto de la oferta de formación brindada por las universidades peruanas dirigido al otorgamiento de una diplomatura en gestión y administración de cooperativas, brindada por la Facultad de Ingeniería Administrativa e Industrial de la Universidad Inca Garcilaso de la Vega en coordinación con la Confederación Nacional de Cooperativas del Perú.

Es necesario mencionar de manera complementaría que en la última década se ofrecieron programas de formación por los propios movimientos de la economía solidaria y las cooperativas (de ahorro y crédito, de servicios, trabajadores, etc.). No obstante, esta oferta se desvinculó de las universidades, era dispersa, fragmentada, carente de continuidad, no tenía identidad, lo que que conllevaba a una constante fuga de cuadros técnicos hacia otras modalidades empresariales. Esto puede ser sintetizado en la declaración de un viejo activista cooperativista cafetalero y actual gerente de la Junta Nacional del Café, Lorenzo Castillo, citado en el trabajo anteriormente mencionado: "Hoy las universidades forman gerentes para negocios urbanos, no para empresas rurales y mucho menos para cooperativas" (2012, p. 338).

\section{Situación de las universidades}

El escritor Alfredo Bryce decía que la Universidad Nacional Mayor de San Marcos (UNMSM) es el "pulmón del Perú", porque a través de esta el país respiraba. Una de las razones que inspiró a Bryce para escribir esta frase era el hecho que durante fines de los cincuenta y comienzos de los sesenta, periodo en el que hizo sus estudios universitarios, ingresaban a esta casa de estudios jóvenes de diferentes regiones del país, convirtiéndola en un crisol de la diversidad existente en el Perú. El propio premio Nobel de literatura, Mario Vargas Llosa (1976), también resaltaría los diversos aprendizajes obtenidos como resultado de sus años de estudiante en esta universidad a fines de los cincuenta y los compartiría a través de los testimonios de varios de sus personajes. Resuenan aún los pensamientos de Zavalita, el protagonista legendario de la novela Conversación en la Catedral, recordando su juventud en San Marcos: cómo había participado en los grupos clandestinos en contra de la dictadura y cómo había caído preso días después de que la Federación de estudiantes apoyara una huelga en respaldo de los ferrocarrileros: "Zavalita se había jodido en San Marcos, pero eso era lo mejor que le había pasado, porque, en el Perú, los que no estaban jodidos eran los que jodían, y él se negaba a formar parte de estos últimos" (p. 45).

Sin embargo, todo este imaginario sanmarquino ha dejado de ser lo que era. Esta universidad no es la única que recibe estudiantes de todo el país; existen otras que han emergido en los últimos años, sobre todo privadas, y han implementado estrategias de marketing y ofertas de formación que han conseguido captar una amplia demanda de estudiantes, y que actualmente les permite tener tantos o incluso más estudiantes que la UnMsM. Por otro lado, esta universidad ha incrementado ostensiblemente el reclutamiento de estudiantes de Lima, sobre todo de las llamadas Limas, Sur, Norte y Este, es decir, de los distritos populares del área metropolitana. Esto le da un rostro más urbano y multicultural en lugar de una imagen provinciana y andina.

La UNMSM, a pesar de estos cambios, mantiene intacta en su identidad institucional, su condición de universidad decana de América, por haber sido fundada el 12 de mayo de 1551 y poseer una de las historias institucionales más antiguas del Perú, incluso anterior a la del mismo Estado peruano. A pesar de esto, no es posible ocultar los problemas que padece como resultado de un largo proceso de crisis acumulada, expresada -según López- en la masificación y el abandono estatal, generadores de la mediocrización de la formación académica y profesional:

Aquello incrementó, por el lado de la demanda, la dificultad para incorporarse al mercado de trabajo. Sobre la base de la masificación, la mediocrización y el abandono estatal, la autonomía universitaria se volvió autarquía y el cogobierno devino en anarquía creando un clima propicio para la frustración estudiantil y para la emergencia de ideologías de la desesperanza que culminan en el terrorismo. El movimiento estudiantil se diluyó y fragmentó, la calidad académica decayó, los operadores aparecieron en la 
escena, la corrupción se instaló en la universidad y las autoridades universitarias buscaron perpetuarse en el poder a través de métodos clientelares y mafiosos (López, 2013, p. 17).

Es pertinente señalar que luego de producido el proceso de transición democrática, después de la caída del presidente Fujimori a comienzos del 2000, la situación de las universidades del Perú no varió sustancialmente e incluso se agudizaron muchos de sus problemas. Lynch al analizar el futuro de la universidad peruana empieza señalando la imposibilidad de imaginar un futuro para esta institución:

Actualmente existen tres tipos de universidades: las públicas, las privadas con dueño y las privadas sin fines de lucro. Entonces, el futuro se presenta como un archipiélago desorganizado en donde la riqueza de la relativa productividad de algunas universidades no podrá siquiera compararse con la pobreza y caos de otras. A partir de esta atomización, el futuro solo puede pensarse como sistemas universitarios paralelos, no sé si competitivos. La realidad fragmentada y en muchos casos caótica de la actual universidad peruana no prefigura ningún futuro (Lynch, 2002, p. 299).

Lynch incide además en varios de los problemas anteriormente señalados: mediocridad, clientelismo, masificación sin proyecto universitario, autarquía; pero profundiza en otro igualmente crítico:

La universidad pública ha dejado de producir conocimiento. Con esto no quiero decir que no haya alguna unidad o facultad o un instituto de investigación donde se produzca conocimiento de manera amplia, positiva y creativa. Digo que orgánicamente, institucionalmente, las universidades públicas han dejado de ser una referencia de producción de conocimientos en el país y para poder tener un futuro ellas deben desarrollar una actividad de investigación consistente. Las enormes dificultades materiales que tenemos para investigar, por una parte y, por otra, el escaso impacto que tiene el trabajo de investigación en la realidad nacional en las últimas décadas, nos hace ver la pobreza que habita entre nosotros en ese aspecto (Lynch, 2002, p. 304).

Este contexto de crisis característico de las universidades del Perú permite comprender de manera más integral el entorno de la experiencia que analizaremos. Estamos frente a una institución universitaria pública de gran antigüedad y tamaño. Hay una complejidad de las relaciones dentro y fuera de esta universidad. Sumado al hecho, como indicamos antes, que en el Perú el tema de las economías solidarias no es visible ni mucho menos central en la agenda pública.

El Seminario de Economía Social, Solidaria y Popular de la UnMsm surge el 2012en la Facultad de Ciencias Sociales 9 "con motivaciones y apuestas individuales de docentes de esta Facultad, que convergen y dan pie a un proceso de institucionalización en la universidad" (Pérez, 2014, p. 2).

El Seminario, según la Resolución Rectoral n. 03998-R-14, constituye una instancia de reflexión acción académica de tipo permanente, que congrega a docentes, estudiantes y representantes de movimientos sociales, orientada a tres objetivos: reflexionar y producir conocimientos sobre la problemática de la economía social, solidaria y popular; formar y entrenar en el manejo de instrumentos teóricos y metodológicos relacionados con procesos de intervención para la economía social, solidaria y popular, y apoyar el fortalecimiento de experiencias de gestión local en las cuales las organizaciones sociales, culturales, económicas e instituciones públicas y privadas afirmen procesos de construcción de formas de economía social, solidaria y popular.

El planteamiento teórico de la experiencia se basa en dos vertientes. Por un lado, se tienen una perspectiva ecléctica, que reconoce corrientes de pensamiento diversas como parte de un proceso amplio de discusión e indagación sobre la problemática de las economías sociales y solidarias o del buen vivir. Busca recolectar evidencias respecto a las relaciones económico sociales, culturales y políticas con la naturaleza de las cooperativas, comunidades y pueblos originarios, redes de productores de comercio justo y agroecológicos, asociaciones de artesanos, bancos

9 La UNMSM está organizada por facultades y no ha incorporado en su discurso académico institucional la superación de la compartamentalización y especialización por campos disciplinarios. La UNMSM posee actualmente 20 facultades y 62 carreras profesionales de grado, 28 doctorados y 96 maestrías, fuera de diplomaturas y segundas especializaciones. Tiene, según información de la Asamblea Nacional de Rectores (2011), una población de 28.645 estudiantes de grado, 53.108 postulantes en promedio compiten cada año por 5.781 vacantes. 
comunales, comedores populares autogestionarios, entre otros.

Por otro lado, está el aporte histórico estructural de las reflexiones pioneras de Quijano sobre el "polo marginal”, realizadas a fines de los años sesenta. Su planteamiento era que Marx registró las tendencias centrales de la problemática de la sobrepoblación relativa o ejército industrial de reserva; pero que estas adquirían contemporáneamente mayores niveles de contradicción. El término "marginalidad", en el planteamiento de Quijano (1977), se refiere, en este sentido, a una nueva dimensión de la problemática de la sobrepoblación relativa. Esta noción surge de una perspectiva crítica sobre el capitalismo en Latinoamérica, porque lo que está en el centro de su planteamiento es qué ocurre con la fuerza de trabajo y los trabajadores que no logran vender su fuerza de trabajo al capital, y, especialmente, qué actividades emprenden, cómo las organizan, y también qué territorios las albergan. No es difícil deducir que su reflexión va más allá de la crítica al crecimiento económico y la modernización capitalista, ya que indaga acerca de las bases mismas de constitución del capitalismo latinoamericano, en las relaciones que lo sustentan, y demuestra que sus posibilidades reales de crecimiento y aporte a la modernización de la sociedad tienen debilidades estructurales innatas que lo limitan.

Quijano (2011b) precisa al respecto, años después, que los esfuerzos de los trabajadores "marginalizados" por resolver sus problemas de supervivencia $y$, en consecuencia, por motivaciones de eficacia práctica, se han ido orientando en muchos casos, cuyo número parece ir en aumento, a fortalecer y a expandir las relaciones económicas de reciprocidad o de intercambio de fuerza de trabajo; a organizarse colectivamente de modo "comunal", en lugar de empresarial, para gestionar adecuadamente sus recursos, sus productos, sus relaciones en el mercado y con los otros sectores tanto de la economía como de la sociedad. Lo que caracteriza la economía popular es justamente que las relaciones de trabajo y de distribución de recursos y del producto se organizan, en lo fundamental, en torno a la reciprocidad y la vida social, las prácticas sociales cotidianas y la comunidad. Eso no significa, por su supuesto, que no esté articulada con el mercado de múltiples maneras y medidas.

Esta reflexión es la segunda vertiente que nutrió la experiencia y sirve de referencia para el proceso de indagación y recolección de evidencia, así como para hacer una intervención aplicada, realizada desde esta. El Seminario fue constituido por el Comité Asesor de la Escuela Académico Profesional de Sociología ${ }^{10}$, gracias a la iniciativa de un grupo de docentes con afinidades en el campo de la investigación en economías sociales, solidarias, populares, comunitarias, y experiencia en la intervención aplicada con cooperativas, comedores populares autogestionarios, bancos comunales, comunidades y pueblos originarios.

Es pertinente anotar que un argumento determinante para convencer a los integrantes del Comité Asesor sobre la pertinencia del Seminario fue la propuesta de establecer alianzas con otras instituciones académicas con el fin de generar instituciones de soporte y animar procesos de investigación acción que contribuyeran a su posicionamiento, así como a revertir su aislamiento. Este aspecto es particularmente sentido por la desarticulación institucional que la Facultad de Ciencias Sociales y sus diferentes escuelas y departamentos afrontan actualmente por la crisis que la afecta.

La presentación de un convenio con la Universidad del País Vasco, que, a través de su Instituto de Estudios sobre Desarrollo y Cooperación Internacional Hegoa, había manifestado su interés de iniciar un trabajo con la UNMSM, terminó de convencer al Comité Asesor para acordar la constitución del Seminario. Sin embargo, la decisión de esta instancia debía pasar a un siguiente nivel en la jerarquía de la universidad: El Consejo de la Facultad de Ciencias Sociales, en el cual también debía recibir una aprobación.

En paralelo, el convenio con la Universidad del País Vasco fue elevado al Rectorado, y, después de varias y largas gestiones, fue aprobado por Resolución Rectoral n. ${ }^{\circ} 03820-R-13$ el 19 de agosto del 2013; pero la aprobación de la creación del Seminario no tuvo igual resultado y demoró un largo periodo.

Un elemento extra es que los representantes de Hegoa, antes de la aprobación del convenio, plantearon un diálogo para evaluar el inicio de acciones conjuntas. El diálogo cedió paso al poco tiempo a la firma de un acuerdo de partes para avanzar en la presentación de un proyecto a la Agencia Vasca de Cooperación para el Desarrollo. Esta propuesta sirvió

10 El Comité Asesor es una instancia de deliberación y toma de decisiones, reúne representantes de docentes y estudiantes de la Escuela elegidos para este fin. Las escuelas académico profesionales son instancias que incluyen a los departamentos y son dirigidas académica y administrativamente por directores elegidos por los consejos de Facultad. El artículo $105 .^{\circ}$ del Estatuto de la UNMSM establece entre otras atribuciones del Comité Asesor: planificar y evaluar el funcionamiento de la Escuela Académico Profesional. 
de aliciente para afianzar no solo la relación entre ambas universidades sino además para dar impulso a la iniciativa de creación del Seminario. La cual si bien venía gestionándose desde antes, logró afianzarse gracias a esta propuesta.

Es interesante hacer notar que de este diálogo surgió la propuesta de cogestionar un programa andino para Perú, Ecuador y Bolivia, el que fue integrado además la Universidad Mayor de San Andrés de La Paz. La actividad principal planteada desde la UNMSM, en el marco de este programa, fue la implementación de un Observatorio de Economía Social, Solidaria y Popular, entendido como una herramienta de recolección de evidencias sobre las economías sociales, solidarias, populares, comunitarias, y que busca aportar al proceso de fortalecimiento de las organizaciones y movimientos sociales vinculados a estas.

Sin embargo, esta acción iría en paralelo al esfuerzo por constituir el Seminario. Es decir, por un lado, se buscó afianzar una instancia institucionalizada dedicada a la investigación acción denominado Seminario de Economía Social, Solidaria y Popular; pero, por otro, gracias al diálogo iniciado con la Universidad del País Vasco, Hegoa, y el apoyo recibido desde la cooperación vasca, fue implementada una acción de gran potencial sinérgico, que permitió articular diversas iniciativas, no solo en el marco del programa elaborado, sino la propia actividad docente de los animadores del Seminario, además de otros docentes, estudiantes y movimientos sociales con los cuales comenzó a establecerse alianzas.

El 15 de noviembre del 2013 fue organizado un taller con representantes de organizaciones y movimientos sociales de la economía social, solidaria, popular, comunitaria, previamente identificados a través de un mapeo, y con los cuales fue desenvuelto un diálogo y reflexión colectiva sobre las relaciones entre la universidad y las organizaciones y movimientos sociales.

El 20 de marzo del 2014 en una ceremonía pública realizada en La Casona de la UNMSM, la sede histórica donde fue fundada esta universidad, fue firmada el acta de constitución del Consejo Social del Seminario y Observatorio de Economía Social, Solidaria y Popular; este se definió como "un órgano colegiado que cumple un rol de asesoría y facilitador del diálogo, la articulación y la cooperación entre la universidad y las organizaciones y movimientos sociales". El Consejo Social está integrado por varias de las principales centrales campesinas y de pueblos originarios del Perú, así como artesanos y productores de comercio justo y redes de economía social y solidaria, entre otras organizaciones figuran: La Confederación Nacional Agraria, la Confederación Campesina del Perú, la Coordinadora Nacional de Pequeños Productores de Comercio Justo del Perú, la Central Interregional de Artesanos del Perú, la Federación Nacional de Mujeres Campesinas, Artesanas, Indígenas, Nativas y Asalariadas del Perú, la Central Café y Cacao, el Grupo Red de Economía Solidaria del Perú, el Grupo Género y Economía.

Este proceso permitió abrir una relación intensa y diversa con varias de estas organizaciones y movimientos, expresada en la implementación de prácticas preprofesionales; la elaboración de reportes de investigación acción, tesis, diseño de planes estratégios y formulación de proyectos de desarrollo en los cursos de las carreras de la Facultad; realización de audiovisuales, actividades de extensión universitaria y proyección social, organización de labores de voluntariado universitario, entre otras.

Paralelamente a la conformación del Consejo Social, la labor de incidencia para lograr la aprobación del Consejo de Facultad de la creación del Seminario fue continuado. Lo paradójico fue conseguir su aprobación en medio de la crisis institucional de la universidad, agudizada de manera extrema entre el 2010 y el 2011; en el caso particular de la Facultad de Ciencias Sociales esto se ve en que no se cuente con un decano debido a las tensiones generadas a partir de las disputas y correlaciones fuerza establecidas entre los diferentes grupos docentes y estudiantiles, incluidas las autoridades de la propia universidad, rector y vicerrector de Investigación, que jugaron un papel determinante en su crisis institucional. Esto desemboca en el nombramiento de un decano encargado desde el Rectorado, que intenta contener las tensiones; sin embargo, en la práctica esto simplemente prologa la crisis y somete a la Facultad a una situación de precariedad institucional antes no conocida. Un dato que ilustra esta precariedad es que durante los últimos cuatro años la Facultad ha tenido cinco decanos.

Esta situación determinó que los decanos encargados, en mayor o menor medida, tuvieran la necesidad de redefinir sus coaliciones y correlaciones de fuerza, es decir, establecer con cuidado su política de alianzas, porque el panorama era extremadamente precario e incierto. Esto indirectamente favoreció la propuesta de creación del Seminario y su posterior institucionalización, porque cuando fue planteada recibió el respaldo de las autoridades. No es arbitrario 
deducir que una lógica pragmática interesada en mostrar resultados en medio de la precariedad primó en las decisiones de las autoridades encargadas y por ello, sobre todo los dos últimos, brindaron su respaldo a la iniciativa.

También es necesario mencionar que las organizaciones juveniles universitarias elegidas como representantes, a través del Tercio Estudiantil en el Consejo de Facultad, jugaron un papel importante al apoyar la aprobación de la iniciativa.

El resultado de este proceso fue la Resolución de Decanato n. ${ }^{\circ}$ 0318-D-FCCss-2014 del 10 de abril del 2014, que aprueba la creación del Seminario; esta meses después fue seguida por la Resolución Rectoral n. ${ }^{\circ}$ 03998-R-14 del 6 de agosto del 2014, ratificando su creación.

Este largo proceso de incidencia interna y de vinculación con organizaciones y movimientos sociales fuecomplementado con la actividad principal del programa cogestionado con Hegoa: la implementación del Observatorio. El resultado fue el diseño del portal web del Observatorio y su alojamiento en el servidor de la universidad, en la dirección: http://economiassolidarias.unmsm.edu.pe

Es necesario anotar que la creación del Observatorio fue resultado de un proceso participativo a través del cual fue consultado su diseño con las organizaciones y movimientos sociales integrantes del Consejo Social. Además sus miembros fueron capacitados en el manejo y gestión de esta herramienta. El uso de tecnologías de información y comunicación fue valorado por las propias organizaciones y movimientos como un recurso potente para su fortalecimiento.

La experiencia del Seminario, más allá de la descripción del proceso de incidencia desplegado dentro de la UNMSM, reúne, desde nuestra perspectiva, tres cuestiones necesarias de evaluar para el afianzamiento de la experiencia: la reproducción de posibles asimetrías en las relaciones entre organizaciones y movimientos con la universidad, la dependencia de la cooperación internacional y el afianzamiento de relaciones institucionalizadas hacia dentro de la universidad.

Respecto a la primera cuestión, es pertinente precisar que muchas de las actividades realizadas desde el Seminario con las organizaciones y movimientos no han logrado del todo basarse en un diálogo de saberes, porque en más de un caso se reproducen las jerarquías entre los saberes de la universidad y los de las organizaciones y movimientos a través de las prácticas de docentes, estudiantes e integrantes de organizaciones y movimientos. No es que no exista una apuesta clara por superar esto desde el Seminario; todo lo contrario, la preocupación por superar estas jerarquías es permanente y está presente. Sin embargo, la realidad es que se sigue reproduciendo en el trato cotidiano y en la realización de las actividades. El hecho de que estas actividades estén sometidas, a través del Consejo Social, a un mecanismo de evaluación permanente es uno de los pocos recursos al cual apelar para lograr de manera efectiva la superación de esta situación. El reto es afianzar este mecanismo de diálogo entre la universidad y las organizaciones y movimientos, para fortalecer relaciones paritarias y evitar la reproducción de posibles asimetrías. El despliegue de un diálogo de saberes no constituye al final de cuentas una declaración de buenas intenciones, sino una práctica real nutrida de la vida social y, por lo tanto, de relaciones de poder necesarias de ser evidenciadas constantemente para afianzar un trato cotidiano más democrático.

La segunda cuestión es que el Seminario ha financiado sus actividades con fondos de cooperación internacional. $\mathrm{Su}$ acceso a estos no ha sido amplio pero sí ha representado un recurso clave para dar impulso inicial a su accionar. Esto debido sobre todo a la crisis de la universidad, que no le ha permitido brindar financiamiento y no tampoco gestionar recursos provenientes de políticas y programas públicos o de agentes locales o internacionales. El establecimiento de alianzas estratégicas Sur-Norte constituyó en este sentido el medio principal a la que apeló para desenvolver sus actividades. El interrogante que surge es en qué medida el Seminario es dependiente de esta estrategia e incluso hasta dónde el uso y acceso a los recursos de la cooperación dejan de ser una estrategia o medio para convertirse en un fin en sí mismo. Sin lugar a dudas este asunto constituye uno de los desafíos mayores que tendrá para los próximos años. No tanto porque su acceso a los fondos de la cooperación internacional crezcan demasiado, a final de cuentas el Perú no constituye una prioridad actual de las financieras y agencias de cooperación internacional, lo que explica la reducción de estos en los últimos años, sino porque la actividad del Seminario puede hacerse dependiente de una sola fuente de recursos. Tal vez la combinación de fuentes de financimiento es un aspecto clave para considerar; la presentación sostenida de proyectos a fondos de investigación de la propia universidad, por más limitados, exiguos o complicado que sea acceder a ellos, o la gestión ante fuentes de 
financiamiento público sobre todo dirigidos a ciencia y tecnología deberían ser asuntos prioritarios de un razonamiento interesado en preservar la autonomía económica y evitar la dependencia de los fondos de cooperación internacional.

Sin embargo, una tercera cuestión igual de importante tiene que ver con el afianzamiento de relaciones institucionalizadas hacia dentro de la universidad. Desde su inicio el Seminario ha intentado ligarse a los cursos de las carreras conducentes al grado y de ahí construir procesos de producción de saberes o intervenciones aplicadas de la mano con actividades de docentes y estudiantes. Este nexo ha ligado su intervención a las actividades desenvueltas en departamentos y escuelas de la universidad. Esta relación hacia dentro y principalmente con los procesos de formación de grado ha contribuido a afianzar su entronque con el entrenamiento académico, porque anima además de labores de formación, tareas de investigación, así como experiencias de extensión universitaria y proyección social. No están del todo consolidadas estas relaciones, pero es evidente que su despliegue constituye un fuerte animador de actividades académicas.

La relación con el proceso de formación de posgrado constituye más bien un vacío, al no haber logrado aún vincularse con su accionar. No es posible desconocer que los procesos de formación a nivel de posgrado constituyen un campo estratégico para la producción de saberes y la incidencia en la comunidad académica, necesario de trabajar a través de una oferta diversificada que contemple los estudios de doctorado, de maestría y diplomados, dirigidos a posgraduados.

Tampoco ha sido posible aún realizar actividades que involucren a los trabajadores no docentes de la universidad. Han sido realizadas algunas actividades, principalmente ferias de productores y campañas de comercio justo, que permitieron a los trabajadores no docentes participar sobre todo como consumidores; pero no ha sido tejido un nexo más sistemático para articular las labores del Seminario a la actividad de este estamento de la comunidad universitaria y que potencialmente podría permitir a la universidad dotarse de una política de responsabilidad social desde enfoques como los de la economía social, solidaria, popular, comunitaria. Además de desenvolver iniciativas innovadoras como el fomento del consumo ético de la mano con los pequeños productores de las redes de comercio justo, agroecológicos, cooperativas, comunidades y pueblos originarios, etc.

\section{Trama compleja}

La experiencia examinada muestra el desenvolvimiento de manera germinal y con todas sus limitaciones de un esfuerzo encaminado a generar diálogos de saberes. No comparte el supuesto de desconocer los saberes producidos por organizaciones y movimientos sociales, invisibilizarlos o asignarles un nivel inferior en una supuesta jerarquía de conocimientos. a experiencia brinda insumos para realizar una crítica de la perspectiva aún hegemónica, en muchas universidades, respecto al divorcio de propuestas como el buen vivir con la sociedad en general, con los productores, así como con las organizaciones y los movimientos sociales.

No podemos afirmar que en la experiencia analizada haya una decisión por entrar en disputa con la perspectiva hegemónica en muchas universidades, expresada en la desvinculación con la sociedad, las organizaciones y movimientos sociales, y los saberes producidos desde sus prácticas. Tal vez incluso desde las experiencias consideradas no se dé esta necesidad, porque su fundamento si bien tiene un soporte teórico metodológico, no parte de la necesidad de resolver un problema exclusivamente académico, sino más bien desplegar una experiencia viva, nutrida de relaciones sociales cargadas de un imaginario social sustentado en vinculaciones tejidas con organizaciones y los movimientos sociales.

A pesar de esto, la experiencia analizada brinda una opción diferente que muestra un esfuerzo sostenido y hasta un camino recorrido posible de replicar en otras realidades, no necesariamente reproduciendo fielmente su experiencia, pero sí recogiendo algunos de sus aprendizajes e incluso asumiendo algunas de las herramientas empleadas en su proceso de autogestión y cogestión.

Esta experiencia, por lo tanto, muestra que el canon epistemológico universitario eurocéntrico no es el único modelo de referencia para la producción de saberes y conocimientos. La experiencia aporta una práctica teórica aplicada sustentada en un proceso dinámico y de gran complejidad, no reducida al ámbito de las dinámicas de las propias universidades, sino enhebradas con otros actores, como los movimientos sociales y la cooperación internacional.

Entonces, frente a la pregunta " $i$ existen experiencias que muestren evidencias de vinculaciones diferentes?" la respuesta es que vienen emergiendo de manera germinal esfuerzos necesarios de consolidar, 
en los cuales las relaciones entre economías solidarias y universidades latinoamericanas, como en el caso de UNMSM, exhiben formas de vinculación que no comparten, necesariamente, el canon epistemológico eurocéntrico, y despliegan más bien iniciativas orientadas a ir más allá de este, lo que proporciona un protagonismo a los saberes producidos desde las vidas de los productores, así como de sus organizaciones y movimientos sociales. Estos esfuerzos, que se muestran en la experiencia examinada, exhiben también apuestas éticas, pasión romántica y la voluntad necesaria para desenvolver una vinculación diferente entre economías solidarias y universidades, ingredientes necesarios en todo proceso histórico social humano que apueste por el buen vivir.

\section{Epílogo sin final}

Como señalaba Coraggio, es preciso que haya sujetos colectivos comunitarios y sociales que planteen sus necesidades de formación, conocimiento y servicio en un diálogo institucionalizado, pero abierto a la innovación y al aprendizaje para permear al conjunto de la universidad. También es necesario que existan actores universitarios que busquen recoger estas necesidades y posean la sensibilidad suficiente para tejer estas relaciones dialógicas institucionalizadas, igualmente abiertas a la innovación y el aprendizaje.

Estos actores universitarios, igual que los sujetos colectivos comunitarios -señalados por Coraggio-, elaboran prácticas y discursos que pueden permear a la universidad. No son, por lo tanto, prescindibles de una lectura de conjunto de los procesos analizados y muchos menos pueden estar ausentes de procesos de intervención como los descritos a través de la experiencia de la UNMSM.

Por lo tanto, además de los sujetos colectivos comunitarios, es necesario reconocer a los actores universitarios interesados en realizar experiencias de vinculación entre economías solidarias y universidades. Es preciso incluso realizar estrategias de fomento y fortalecimiento para estos actores en la medida en que pueden resultar claves en las dinámicas antes evidenciadas.

Un desafío futuro es que la experiencia de las relaciones entre economía solidarias y universidades, que reúnen a sujetos colectivos comunitarios y sociales con actores universitarios interesados en desenvolver estas experiencias, no constituyan esfuerzos aislados y circunscritos a las fronteras nacionales o ámbitos localizados, sino comiencen a enhebrarse a través de redes de escala regional y global, donde sus anclajes territoriales y las experiencias país desenvueltas puedan compartirse y ponerse en diálogo. Este proceso adquiere un peso determinante al menos si realmente se busca permear de manera efectiva a las universidades y consolidar las relaciones tejidas con las organizaciones y movimientos de las economías solidarias y el buen vivir.

Resulta indudable que estas relaciones pueden ser vistas a través de muchas otras historias, como la aquí contada; pero la experiencia analizada consideramos es ilustrativa de estas relaciones actuales, y que desde la presente narración ha intentado ser retratada, no como parte de un relato épico o una oda idíli$\mathrm{ca}$, sino simplemente como la expresión de esfuerzos desplegados desde la vida cotidiana de las y los productores de las economías solidarias, junto a sus organizaciones y movimientos, y las y los universitarios de la Latinoamérica de hoy.

\section{Referencias}

Asamblea Nacional de Rectores. (2011). Datos estadísticos universitarios. Lima: Dirección de Estadística de la Asamblea Nacional de Rectores.

Acosta, M. y A. Levin. (2013). La formación universitaria en cooperativismo y economía social en Argentina. Buenos Aires: Centro de Estudios de Sociología del Trabajo/ Facultad de Ciencias Económicas/ Universidad de Buenos Aires.

Cárdenas, G. (1983). El sector de economía social en el Perú. Cooperativas y empresas autogestionarias. Lima: Centro de Estudios para el Desarrollo y la Participación.

Castro-Gómez, S. (2007). Decolonizar la universidad. La hybris del punto cero y el diálogo de saberes. En S. Castro-Gómez y R. Grosfoguel (comp.), El giro decolonial. Reflexiones para una diversidad epistémica más allá del capitalismo global (pp. 79-91). Bogotá: Iesco/ Pensar/Siglo del Hombre.

Coraggio, J. (2001). Construir universidad en la adversidad. Desafíos de la educación superior en América Latina. Presentación en la II Reunión de Ministros de Educación de las Américas en el Ámbito del Cidi, Punta del Este, 24 y 25 de septiembre.

Coraggio, J. (2002). Universidad y desarrollo local. Ponencia presentada en el Seminario Internacional: La Educación Superior y las Nuevas Tendencias, organizado por Conesup, Unesco, Ciespal, Quito, 23 y 24 de julio.

Coraggio, J. (2011). La economía social como vía para otro 
desarrollo social. En A. Acosta y E. Martínez (Comp.), José Luis Coraggio. Economía Social y Solidaria. El trabajo antes que el capital (pp. 43-68). Quito: Abya Yala.

Coraggio, J. (2014). La economía social y solidaria: el papel de las universidades. Ponencia presentada en el seminario: Universidad Pública y Economías Solidarias, organizado por el Seminario de Economía Social, Solidaria y Popular, Facultad de Ciencias Sociales, Universidad Nacional Mayor de San Marcos y el Instituto Hegoa de la Universidad del País Vasco, Lima, 9 de diciembre.

Cruz, A. (2004). É caminhando que se faz o camino: diferentes metodologias das incubadoras tecnológicas de cooperativas populares no Brasil. Cayapa. Revista venezolana de Economía Social, 4(8).

García, A. (2007). Universidad y educación cooperativa en Venezuela: experiencias. En Universidad y educación cooperativa en Venezuela, memorias del Primer Coloquio Nacional "Universidad y Educación Cooperativa”, organizado por el Centro Interdisciplinario de Investigación, Formación y Documentación de la Economía Cooperativa, Social y Pública, Universidad de Los Andes, Trujillo, del 31 de mayo al 02 de junio.

Germaná, C. (2013). Iniciativas universitarias para la economía social y solidaria en el Perú. Ponencia presentada en el seminario Derechos económicos y políticas públicas de economía social y solidaria: retos para la universidad desde el suma qamaña. Universidad del País Vasco-HEGOA y la Universidad Mayor de San Andrés-CIDES, La Paz, del 17 al 19 de enero.

Izquierdo, M. (2009). Problemas de las empresas cooperativas en México que atentan contra su naturaleza especial. Boletín de la Asociación Internacional de Derecho Cooperativo, 43, Bilbao: Asociación Internacional de Derecho Cooperativo.

Leher, R. (2010). Capitalismo dependiente y educación: propuestas para la problemática universitaria. En R. Leher (Comp.), Por una reforma radical de las universidades latinoamericanas. Rosario Santa Fe: Consejo Latinoamericano de Ciencias Sociales, Homo Sapiens Ediciones.

López, S. (2013). Acción universitaria para refundar San Marcos. En Por un acuerdo para refundar la Facultad de Ciencias Sociales y la Universidad de San Marcos. Lima: Facultad de Ciencias Sociales, Universidad Nacional Mayor de San Marcos.

Lynch, N. (2002). El futuro de la universidad peruana", en Aljovín, C. y C. Germaná (eds.), La universidad en el Perú, Lima: Universidad Nacional Mayor de San Marcos.
Mato, D. (2011). Universidades indígenas de América Latina: logros, problemas y desafíos. Revista Andaluza de Antropología, 1.

Mogrovejo, R., Vanhuynegem P. y Vásquez, M. (2012). El renacimiento del modelo cooperativo peruano. En R. Mogrovejo, P. Vanhuynegem y M. Vásquez (Eds.), El cooperativismo en América Latina. Una diversidad de contribuciones al desarrollo sostenible. La Paz: Organización Internacional del Trabajo.

Padilla, A. (2008). Las universidades indígenas en México. Inclusión o exclusión. Veredas, número especial, primer semestre.

Pastore, R. (2010). Un panorama del resurgimiento de la economía social y solidaria en Argentina. Revista de Ciencias Sociales, 2(18).

Pérez-Alva, E. (2014). Bordando la vida desde otras economías. Ponencia presentada en el Encuentro de vinculación socioeconómica, desarrollo socio-técnico e incubadoras universitarias. Universidad Nacional de Quilmes, Buenos Aires, 21 y 22 de noviembre.

Quijano, A. (2011a). "Bien vivir": entre el "desarrollo" y la des/colonialidad del poder". Ecuador Debate, 84.

Quijano, A. (2011b). ¿ ¿Sistemas alternativos de producción? En B. Santos (Coord.), Producir para vivir. Los caminos de la producción no capitalista. México: Fondo de Cultura Económica.

Quijano, A. (1997). Colonialidad del poder, cultura y conocimiento en América Latina. Anuario Mariateguiano, 9.

Quijano, A. (1977). Imperialismo y "marginalidad" en América Latina. Lima: Mosca Azul Editores.

Rénique, J. (1987). De la fe en el progreso al mito andino: los intelectuales cusqueños. Márgenes, 1.

Rofman, A. (2005). Introducción. En A. Rofman (Comp.), Universidad y desarrollo local. Aprendizajes y desafíos. Buenos Aires: Universidad Nacional de General Sarmiento y Prometeo Libros.

Singer, P. (2011). La reciente resurrección de la economía solidaria en Brasil. En B. Santos (Coord.), Producir para vivir. Los caminos de la producción no capitalista. México: Fondo de Cultura Económica.

Vargas-Llosa, M. (1976). Conversación en la Catedral. Madrid: Seix Barral. 